\title{
DUNGIA ET LE MOBUTISME. COMMENT DIRE LES SECRETS DE LA CORRUPTION ?
}

\author{
Abderrahman BEGGAR, Wilfrid Laurier University
}

\section{Résumé}

Le propos de cet article est d'étudier les modes d'énonciation du secret de la corruption à partir du témoignage d'Emmanuel Dungia dans Mobutu et l'argent du Zä̈re. Révélations d'un diplomate, ex-agent des services secrets. Ce récit est considéré à partir de son inscription dans un discours de contre-pouvoir accompagnant le déclin du régime de l'ancien dictateur.

Le propos est d'analyser les diverses stratégies adaptées par l'auteur dans son désir d'assurer une certaine autorité à son récit, notamment comment derrière la confession et le voyeurisme se cache le besoin d'affirmer une certaine autorité.

\section{Introduction}

Mobutu et l'argent du Zä̈re. Révélations d'un diplomate, ex-agent des services secrets se définit comme un «témoignage de première main sur la cause de la ruine morale et économique » de ce pays africain (voir la quatrième de couverture). Le propos du livre, y lit-on, est de « satisfaire la curiosité du lecteur » (76) en lui révélant des vérités cachées. Aux yeux de son auteur, Emmanuel Dungia, ce qui lui donne force c'est surtout sa nature de témoignage à partir du lieu privilégié que lui procuraient ses fonctions:

Diplomate et officier de renseignements du principal Service de Sécurité du Zaïre, héritier de la Sûreté publique coloniale, j'ai donc été successivement affecté de 1980 à 1989 à Paris, à Bruxelles et à Rome auprès du Saint-Siège. À mon retour au pays, en 1989, j'ai exercé les fonctions de Conseiller politique et diplomatique de Nguz à Karl-I-Bond, Ministre des Affaires Etrangères. Ces postes ont été des observatoires exceptionnels à partir desquels j'ai pu glaner fortuitement des renseignements forts utiles. (13)

Sans perdre de vue le contexte historique, cet ouvrage s'inscrit dans un discours de résistance. Son auteur témoigne de l'agonie politique de Mobutu à une époque où il commence à avoir le titre de «léopard de papier » (Lanotte 22) ou de «paria de luxe » 
(Braekman 35). À part sa valeur de témoignage, d'un point de vue personnel et original, nous tenons à préciser que ce document traite d'un sujet connu, la corruption au temps de Mobutu étant l'un des sujets les mieux étudiés. Aussi notre but n'est-il pas de partir de ce livre pour faire l'anatomie de la corruption, ni d'en évaluer la valeur historique, ni de lui assigner une place dans un corpus déjà exhaustif. L'objectif n'est pas non plus de répondre à la question : «qu'est-ce que la corruption ?», mais de voir comment l'on parle de la corruption. Surtout, comment le discours anti-corruption s'inscrit dans une stratégie de résistance et se constitue en contre-pouvoir. Notre approche s'appuie sur deux fondements.

\section{Secret et pouvoir}

Partant des travaux de Benveniste qui stipule que toute prédication obéit à la volonté de s'affirmer par le biais du langage, Coquet parle de la «modalité du métavouloir ». Cette modalité définit ce qu'il qualifie d' «infrastructure » du discours qui n'est autre que l' «histoire individuelle de celui qui parle»(16). Le sujet énonçant s'affirme en énonçant, et cet acte prime l'existence de ce dont il parle. En parlant de Mobutu et du mobutisme, Dungia ne fait qu'affirmer son pouvoir en tant qu'énonciateur de secrets. Cette affirmation ne peut se réaliser que par rapport à autrui. Elle est liée directement à une situation de contact, d'affrontement : «Je m'affirme vis-à-vis de moimême et aussi vis-à vis d'un autre ». C'est cette situation de renvoi qui est le fondement de tout acte d'énonciation. De manière explicite ou implicite, tout énoncé obéit à cette nature dialogique (Bakhtine) : même dans une situation monologique, quand le destinataire est soi-même, l'énoncé porte les stigmates de la présence d'autrui, du simple fait que parler suppose le respect de normes linguistiques, elles-mêmes tributaires du conventionnel et du collectif. Par conséquent, l'affirmation de soi repose sur cette nature relationnelle. Une telle situation suppose des rapports hiérarchiques. La «logique des places » prend vigueur de manière particulière dans le discours sur le secret étant donné le pacte de lecture suppose une énonciation à partir d'un lieu privilégié et que celui qui rapporte est là pour combler des désirs et non pour vérifier des hypothèses.

La fonction scriptuaire consiste à inscrire le dire dans un discours dont le savoirpouvoir est doté de la capacité de pénétrer les sphères privées du pouvoir en place ${ }^{1}$. Les 
témoignages qu'il contient, portent surtout sur les structures répressives au Zaïre de Mobutu $^{2}$. Qui dit structure policière, dit mondes protégés par la loi du secret, inscription dans un espace non partagé (la racine latine «secretus » signifie «placé ailleurs»). Le secret est d'abord un lieu dont la conception dépend de celui qui l'énonce. Il émane d'une stratégie de représentation donnée et constitue une valeur ajoutée qui inscrit le fait dans un lieu porteur d'un sens déterminé. C'est pour cette raison qu'il est à la base du pouvoir. Pour Bertrandias,

le secret est à la source du pouvoir ; le savoir comme le sacré prétendent détenir le grand secret, exalté, inviolable, désiré de tous ; on secrète du secret, on cache pour montrer que l'on cache, et on se cache pour être trouvé. Être socialement, c'est se placer au centre du secret. (5)

Plus qu'autre chose, le secret relève d'une mise en scène. Celle-ci détermine le reste au point que le référent devient prétexte et la vérité un souci secondaire. Le pouvoir est présenté comme ce qui se cache derrière un voile de discrétion ${ }^{3}$. C'est pour cette raison que chez Mobutu, le caractère le plus saillant est son «don extraordinaire de dissimulation » (14), une particularité largement partagée par l'entourage du maréchal. Ramener le secret au terrain du partagé revient à ouvrir le pouvoir à la conquête de l'œil commun. En rejoignant le terrain du visible, le secret intègre la sphère du social. Cette intégration le neutralise et le dépouille de sa transcendance ${ }^{4}$.

Le livre regorge d'anecdotes sur l'entourage de Mobutu, un milieu composé de cette catégorie d'individus que Duke qualifie de « predator class » (151), des membres de l'oligarchie militaire, civile (surtout financière) et de la nomenklatura ethnique et familiale ${ }^{5}$. Plus que de satisfaire un besoin de justice, ces anecdotes servent à assouvir un désir voyeur. La quantité de détails sur l'intime livre les zones interdites, le voilé et le tabou au commun et en banalise la portée. Le récit se veut sui generis dans la mesure où il est le seul garant de la véracité de ce qu'il met en scène ${ }^{6}$. L'autosuffisance se voit dans l'autonomie des contenus sachant que le désir de voir l'emporte sur celui de vérifier.

\section{Une quête de puissance}

En effet, la volonté de s'affirmer, qui anime tout acte énonciatif et lui assure son caractère dynamique, a beaucoup en commun avec une notion de Nietzsche, pour qui, derrière tout phénomène, il y a une constellation de forces qui s'approprient un objet et 
lui donnent sens. Au lieu d'interprétation, il vaut mieux parler de «symptomalogie ». Dans cette logique, le discours sur la corruption ne doit pas être dissocié des stratégies propres au pouvoir. Comme discours de résistance, il véhicule des vérités qui à leur tour obéissent à un certain agenda. La «vérité »n'est pas considérée en tant qu'a priori, quelque chose de neutre en attente de confirmation ou consolidation, mais plutôt dans son rapport ontologique au pouvoir qui en est l'incarnation et qui, de ce fait, se charge de la diffuser à travers les divers corps sociaux (Foucault). La stratégie du discours sur la corruption consiste à apporter des vérités qui en minent d'autres en vue d'établir un nouvel ordre. Ces vérités s'arrogent un pouvoir qui réside surtout dans l'acte même de leur révélation.

Quand écrire est contre-pouvoir, l'impératif premier n'est pas forcément le contenu, mais le fait de rendre public le mécontentement. Vient ensuite le souci de doter son discours d'autorité. Le récit est parsemé d'énoncés du genre : «En ma qualité de Conseiller politique et diplomatique »(21), «[En] 1977, possibilité m'est donnée de découvrir son [Mobutu] penchant excessif pour le luxe et la jouissance qui va provoquer la ruine économique du pays » (29), «Une des personnalités qui ont pris part à l'heureux évènement me confiera que ce jour-là, il a eu pitié du Zaïre » (37). Ces déclarations rendent explicite le besoin d'auto-affirmer l'autorité de l'énonciateur et, par conséquent, d'imposer une image capable d'assurer crédibilité au discours. Pour que cette dernière ne paraisse pas comme autoproclamée, elle doit donner preuve des processus qui l'assurent. Ceux-ci obéissent à deux mouvements, l'un endogène, où Je se définit lui-même, et l'autre exogène, où il fait appel à l'autre. La lecture sert à mettre entre elles les pièces éparses d'une vraie imago-genèse : en informant sur la corruption, le témoin accouche progressivement de sa propre image. Celle-ci s'actualise tout au long de la narration et est prise en charge par une diversité de voix : le sujet énonçant (Dungia) ainsi que les divers sujets (diplomates étrangers, religieux, hauts fonctionnaires, hommes politiques et autres) qui viennent ajouter leurs témoignages. Ces voix ne donnent pas de témoignages directs ; leurs portraits se dégagent de leurs réactions face à l'auteur. Ainsi, l'autorité se voit confirmée par leur confiance en lui. La qualité la plus récurrente est celle de la confiance (le sujet est couronné d'autorité par ses pairs, qui viennent lui confesser leurs déboires dans les dédales de l'administration mobutiste), pour leur besoin de son 
expertise (il est consulté dans des affaires de prime importance allant de la sécurité aux finances), par leur appel de son sens de la justice (il est invité à donner son avis sur des matières relevant de l'ordre juridique).

Le texte s'organise à partir de regards croisés. Pourtant, l'hétérogénéité énonciative s'estompe devant l'unicité du projet narratif, qui est d'assurer que la parole de l'autre soit acceptable et de donner du pouvoir à son savoir.

Ces sujets-témoins sont réduits à la fonction qui leur est léguée. Ils se caractérisent par une uniformité qui réduit leur densité et consistance. La contradiction est une caractéristique totalement absente parce que l'auteur ne prend pas le temps de décrire ses interlocuteurs, de considérer leur profil psychologique, de les mettre dans un contexte propre, de nous présenter des êtres en chair et en os. Cette réduction fonctionnelle se traduit par un manque de clarté idéologique : ce sont des sujets présentés souvent comme apolitiques, même s'ils sont, dans la plupart, des cas, des acteurs politiques actifs. Et, même si leur orientation politique est présente (les opposants), elle ne joue aucun rôle significatif dans la définition de leur identité ; le politique est cité juste à titre indicatif de l'importance hiérarchique ou contestataire.

Chaque témoignage s'inscrit dans une stratégie d'élaboration identitaire permanente. Par cette attitude, l'anti-pouvoir constitue sa propre mythologie. Il s'agit d'un discours contextualisé, inscrit dans un cadre conjoncturel précis (le Zaïre de Mobutu), en même temps que dé-contextualisé (les sujets énonciateurs étant hors de leur réalité historique). Cette deuxième opération sert surtout à couvrir la narration de transcendance. C'est par elle que le dire ne s'inscrit plus dans le fait mais dans l'événement; les propos défont l'éphémère et sont paroles fondatrices destinées à s'inscrire dans la mémoire collective et à donner une assise au discours opposant. Dans ce même esprit, Dungia veut inclure son travail dans une réflexion qui dépasse le Zaïre pour englober la condition humaine. Dès l'introduction, le propos est clair: «Ainsi la dénonciation de toute dictature constitue-t-elle la finalité de ce livre » (12). Le regard de Dungia est placé non seulement dans une contextualité interne (le dedans du pouvoir mobutiste), mais aussi dans une dé-contextualité propre à un dehors idéologique aux allures apolitiques qui lui assure une moralité insoupçonnable. De ce fait, la vérité propre au dedans trouve dans le dehors la couverture d'un jugement para-empirique. La 
polyphonie ne fait que traduire la fragilité et l'indécision qui marquent en général le témoignage. Elle fait partie de l'effort de répondre au besoin de mettre l'histoire personnelle sous l'aile de l'Histoire.

C'est le côté autobiographique du récit, son apparente subjectivité qui fragilise les vérités. Ce doute creuse des brèches dans le corps même du discours, le prive de son homogénéité et de son autorité pour l'ouvrir sur des voix autres, qui viennent restituer le manque d'objectivité propre au travail historiographique. La polyphonie est là pour se substituer à l'enquête. Les témoignages supplantent documents d'archives, rapports, travaux académiques, médiatiques ou autres. La succession de ces témoignages donne à la narration un caractère ressemblant à l'enquête. Cette narration ayant comme fonction dans ce contexte, de même que dans celui du travail historiographique, de répondre au besoin de vérité, une vérité qui transcende le plan factuel pour rejoindre celui de la raison scientifique. D'ailleurs, c'est ce même besoin qui est derrière la présence de documents annexes (120 pages sur les 221 que compte le livre). Ceux-ci sont constitués d'un ensemble d'écrits propres au registre historiographique: rapports, correspondance officielle, factures, relevés bancaires, textes de lois, articles journalistiques.

\section{La dictature concentrationnaire}

Le pouvoir discursif est au service d'une stratégie qui s'appuie sur l'idée que la vérité du mobutisme se révèle partout dans une société où la corruption est le moteur essentiel. Une grande partie du livre est constituée de descriptions d'un monde apocalyptique, d'hôpitaux où les malades sont pris en otages, faute de moyens pour payer, de bidonvilles, d'infrastructures délabrées, d'une société en crise spirituelle et morale : «Depuis les années 70, le Zaïre, tel un navire en perdition, va à la dérive. Le pays présente l'aspect d'une terre en ruines à la sortie d'une guerre ou d'un cataclysme » (25).

Désigner les réseaux de criminalité financière et politique, attirer l'attention du lecteur sur ces archipels d'opulence, en contraste flagrant avec l'océan de misère qui les entoure, sont des manières d'explorer les raisons ontologiques derrière les défaillances des mécanismes du pouvoir. Si l'institution qui fonctionne le mieux est la policière ${ }^{7}$, c'est parce que la mission première que le régime s'est assignée, est d'assurer sa propre survie. 
Pour cette raison, l'État opte pour une stratégie défensive et concentre toute son attention sur la machine policière et le pouvoir inquisitoire du parti unique (le Mouvement populaire révolutionnaire). Ceci est dû au fait que, comme beaucoup de régimes enfantés pendant la Guerre froide pour servir des intérêts majoritairement stratégiques, le mobutisme a du mal à se constituer une base populaire ${ }^{8}$. La réduction de la discipline à son état primaire n'a fait qu'éloigner le gouverné de celui qui le gouverne. À la visibilité du pouvoir s'oppose l'invisibilité de la masse. Le pouvoir manque ainsi du savoir-pouvoir qui lui aurait permis de mieux contrôler, de mieux pénétrer les diverses couches sociales. La discipline n'a pas atteint cet état panoptique dont parle Foucault, où le pouvoir se fait invisible et est partout et nulle part. En février 1991, à bord de son bateau, « après un long séjour à Gbadolite », son village natal, Mobutu arriva à la capitale «pour faire taire les rumeurs des préparatifs de sa fuite à l'étranger ». Ce qui suit, montre clairement comment gouvernant et gouverné évoluent dans des modes différents:

Arrivé au niveau du village de Maluku, proche de la Cité de l'ex-Parti unique à N'Sele, à $50 \mathrm{~km}$ de Kinshasa, il aperçoit sur la rive du fleuve une foule de femmes agitant des foulards et scandant 'Tata ayei, nzala esili !' ce qui signifie à peu près : 'Papa de retour, finie la disette !' Il est aux anges, mais sa joie sera de courte durée. Il ordonne au capitaine de son luxueux bateau, le 'Kamanyola', d'accoster. Lorsque le bateau s'immobilise, en un mouvement réglé comme pour un ballet, les femmes se retournent et relèvent leur pagne pour lui montrer leur derrière, en signe de malédiction! (16)

Conscient du gouffre qui le sépare de la population, Mobutu pourrait servir de figure de proue à ce que Frantz Fanon qualifie de «colonialisme interne ». Comme le colonisateur, il se cache derrière des mécanismes oppressifs qui l'éloignent de la réalité sociale. Dans ce sens, il est décrit comme celui qui s'acharne à se différencier à l'exautorité coloniale ; l'auteur parle d'une «obsession à saisir le moindre prétexte pour croiser le fer avec l'ancienne puissance coloniale » (32). Il cherche par tous les moyens à «rabaisser et impressionner l'ancien tuteur» (33). Cette attitude est derrière le développement de tout un discours sur l'identité zaïroise : Mobutu et l'élite qui le supportait, ont créé un discours identitaire qui a fini par les changer eux-mêmes. Cette transformation débouche sur des « comportements presque enfantins » (33).

Mobutu en vient ainsi à reproduire les stéréotypes coloniaux, notamment celui consistant à juger que pour l'Africain la notion de pouvoir est abstraite, qu'il ne peut la 
comprendre qu'une fois incarnée dans la personne du leader. Le pouvoir est voué à une subjectivité extrême ; comme le résume Nzongola-Ntalaja : «Le pouvoir de Mobutu était absolu au point de faire tout ce que le cœur dictait » (141). Du manque de communication entre le peuple et la présidence zaïroise résulte l'image d'un pouvoir dont les tendances dangereusement extravagantes s'expliquent par une absence totale de rationalité. Dans «La démocratie mis[e] au frigo » (30), les opposants confiés aux «sinistres cachots souterrains de la Cité de l'OUA ou [au] fleuve Zaïre » (30), la population « encasernée et caporalisée », sont en réalité des manifestations de cette incapacité du pouvoir d'évoluer quand, suite à un coup d'État, Mobutu arriva à la tête du pays dans ce qui est qualifié, selon l'auteur, de «mariage forcé » (16). La violence incontrôlable et la corruption ne sont que quelques expressions de l'incapacité du pouvoir de trouver en lui-même matière à se régénérer. N'oublions pas que le sens du mot corruption (« décomposition, pourriture, putréfaction, dépravation, gangrène, perversion, souillure, vice » selon Le Petit Robert) renvoie avant tout à l'incapacité, à la paralysie que provoquent la maladie et le mauvais fonctionnement d'un corps. Dans ce contexte de débâcle sociale, le religieux devient le mode de sublimation d'un quotidien dégradé. Sectes et prêcheurs de toute sorte prolifèrent. Le pouvoir aussi est sous la prise du magico-religieux. L'auteur parle d'un Ministère marabout :

Dans son esprit [celui de Mobutu], le monde est gouverné par plusieurs forces mystiques qui ont chacune une parcelle de pouvoir magique sur les choses et sur les êtres animés [...]. Aucune décision importante du Guide n’est prise sans l'avis de M. Kebé [marabout sénégalais]. (42)

Le maraboutisme est ainsi considéré comme le noyau de l'État, une institution qui accapare «3\% du budget public, l'équivalent du Ministère de la santé ». Les rapports à l'Église catholique sont souvent tendus. Selon Dungia, même les fêtes et noms chrétiens sont attaqués. Pour l'auteur, les raisons de cette hostilité s'expliquent par le fait que l'Eglise a encore un certain pouvoir dans les milieux les plus démunis?.

\section{Mettre à nu la dictature}

Le long de son récit, Dungia garde présente sa mission : s'attaquer à tout ce qui constitue le fondement de la représentation d'un Mobutu jugé, selon l'expression de Dunn, comme «personnification du corps politique» (113). Il s'agit de déconstruire ce 
corps monolithique, ce «sujet homogène » qu'est le dictateur ${ }^{10}$ en le délogeant de sa place mythique pour le soumettre dans sa nudité au regard du lecteur virtuel. C'est plus une radiographie de la personne du dictateur que celle d'un système. Le livre prend souvent l'aspect d'une succession de faits divers où l'anecdotique détermine le « régime de vérité ». La vie privée est la cible privilégiée ; c'est là où le pouvoir est loin de sa propre mythologie, loin des rituels qui le fondent et le perdurent. Pénétrer les sphères privées, c'est éclairer tous ces terrains vierges, protégés de l'indiscrétion, pour les soumettre à un œil déontique qui montre par la même occasion que son pouvoir réside dans son omniprésence.

La collection de renseignements montre combien tout mène à Dungia. L'auteur est au cœur de tout un réseau d'informateurs qui le renseignent sur ce qui se passe alentour du dictateur comme, par exemple, les nombreuses anecdotes sur la jalousie de Madame la présidente face aux conquêtes de Mobutu, à travers couvents, famille proche et hiérarchie militaire. Dans ce désir de détruire la dictature, la rumeur est un pouvoir qui n'a besoin de l'aval d'aucune instance. Son énonciateur est invisible et se confond souvent avec la masse. Quant à son énonciataire, il est, lui aussi, indéfini. La rumeur épouse le hasard. Son contenu ne se dévoile qu'à partir du moment où on se l'approprie. Le message qu'elle contient, est prisonnier de l'imprécis et de l'impersonnel qui l'entourent. Il n'attend qu'une parole dotée d'autorité (dans ce contexte, celle de l'auteur) pour la faire sortir de son existence diffuse. Dungia n'hésite pas à se servir de la rumeur pour introduire la vox populi, inscrivant dans son dire celui d'une masse silencieuse réduite à un espace public muselé :

Des sources convergentes affirment que Madame la Présidente ne serait pas morte à 37 ans, d'une crise cardiaque, selon la version officielle. La mort serait la conséquence de scènes de ménage violentes après la découverte de la liaison de son mari avec Madame Kapelé. (95)

L'autorité (« des sources convergentes ») qui prend en charge la rumeur, reste prisonnière du même indéfini qui fait la rumeur. Et, c'est là que nous pouvons voir comment l'acte d'énonciation est en soi une manière d'affirmer le pouvoir de l'énonciateur Dungia. En reprenant le schéma proposé par Coquet, selon lequel tout énoncé s’organise selon deux niveaux, l'un explicite («il dit que ») et l'autre implicite («il affirme que »), nous pouvons voir comment l'énoncé « des sources convergentes affirment que Madame serait 
assassinée par son mari » implique tout d'abord que le sujet rapporteur de la rumeur «s'affirme comme sujet énonçant, ego » et affirme simultanément un attribut au sujet énoncé : «le mari serait un assassin ». Ce que l'on peut schématiser de la manière suivante :

Manifestation : le mari serait un assassin

Immanence : relation (ego, le penchant homicide du mari)

L'usage du conditionnel n'est que formel. La distance qu'il implique, peut être dépassée parce que, comme dans tout discours à finalité déontique, la mission est de soumettre le factuel au moral. En même temps, le préjugé et la croyance annihilent l'hypothétique. La rumeur agit en nébuleuse, pouvoir non articulé, amorphe, aux règles de fonctionnement imprécises. Du fait de ne pas avoir d'énonciataire précis, ce dire est indifférent à tout pacte, d'où son pouvoir absolu. Sous le charme de la puissance de la rumeur, Dungia oublie souvent que son lecteur virtuel est censé incarner une moralité irréprochable ${ }^{11}$; d'où certaines contradictions profondes quant au fond moral du discours : si d'un côté, comme nous allons le voir, Mobutu donne une image dégradante du Noir et confirme les thèses racistes, de l'autre, l'auteur ne cache pas son adhésion à une vox populi insouciante de cacher ses tendances racistes. Comme dans toute situation de crise, l'étranger est l'objet visé du mécontentement. Chez l'auteur, par exemple, Libanais et Africains de l'Ouest sont la cible favorite comme dans ce passage où il parle du

quartier 'Bon marché' baptisé 'quartier Beyrouth' en raison de la forte densité de Libanais par mètre carré, [qui] ne désemplit pas des 'Ndigari' sobriquet désignant les Ouest-Africains - qui, fraîchement débarqués sans un sou, viennent se restaurer et quémander un fonds de démarrage. (44)

Chez Mobutu, même la couleur de la peau entre dans la définition du caractère et du statut :

Il veut une secrétaire bilingue français-italien. On lui présente une jolie secrétaire métisse, trilingue français-italien-anglais, une Soudanaise d'origine ivoirienne. Il lui fait des avances. Elle claque la porte. Il échoue sur une Sénégalaise de teint foncé aux formes arrondies. En difficulté financière, elle accepte le honteux marchandage. (24)

En plus d'un certain lien raciste entre auteur et dictateur, chez les deux, une contradiction majeure se dégage du rapport à l'ancienne puissance coloniale. Si Mobutu est présenté 
dans une relation œdipienne à l'ancien colonisateur, Dungia de même ne ménage pas ses élans nostalgiques vis-à-vis de la domination belge :

Après 80 ans de colonisation, les Belges sont partis en 1960 sans une opposition opiniâtre. [...] Réseaux routiers denses, infrastructures industrielles imposantes, écoles en grand nombre confiées à des missionnaires, centres de soins de santé couvrant tout le pays. Une indiscutable joie de vivre se lit sur le visage des habitants. (26)

Cette représentation idéalisée du colonisateur par l'auteur est liée à la tendance à sacrifier toute objectivité (qui ignore les morts par millions que la présence belge a provoqués, surtout sous le règne de Léopold II) avec la seule fin de montrer que, dans une logique binaire entre bien et mal, Mobutu monopolise le mal.

\section{Discours sur la corruption et identité}

On peut à partir de la notion de «dictature corrompue » distinguer les modes de subjectivation qui gèrent l'univers individuel et collectif. Conceptualiser la corruption consiste avant tout à en élaborer la topographie sociale. Le phénomène est lié tout d'abord à la notion d'appartenance, qu'elle soit tribale, professionnelle, confessionnelle, nationale ou internationale. Le social se voit articulé selon des matrices imposées par le pouvoir corrompu. Le rapport entre le leader et le peuple se présente sous forme de possession sexuelle : «Maréchal Mobutu Sese Seko Kuku Ngbandu wa za Benga (Le coq de la basse-cour qui ne laisse de côté aucune poule), Père-Fondateur de la nation zaïroise, chef du parti Unique. » Le paternalisme qui détermine l'image de Mobutu («PèreFondateur de la nation ${ }^{12}$ ) est doublé d'ironie. La conscience de soi et des autres se définit à partir du sentiment de honte que ressent le pays face au dictateur. De par sa situation d'expatrié, l'auteur diplomate est exposé aux regards étrangers où l'image honteuse du groupe ne cesse d'être reflétée, comme lui révèle «un important homme d'affaires italien $»$ :

M. le Conseiller, votre Président a accompli un exploit extraordinaire [...], faire d'un pays riche un pays pauvre, ce n'est pas donné à tout le monde ! [...] Enfin, ne vous offusquez pas ! C'est un moment difficile pour votre pays ; ça passera. Le Moyen-âge, ici, ce n'était pas la mer à boire ! C'est l'Histoire qui se répète, voilà tout ! (37) 
Le sentiment de honte dessine les contours du récit. La cause de la ruine du régime Mobutu et sa corruption résident surtout dans son anachronisme ${ }^{13}$. Les références à la fin de la Guerre froide, à la chute du mur de Berlin, à la fin du régime en Roumanie et aux réactions d'une administration étasunienne qui n'en pouvait plus devant les agissements du dictateur sont des leitmotive ${ }^{14}$. Le sentiment de honte est engendré par ce préjugé largement répandu ; l'anarchie qu'a connue le Zaïre dès son indépendance a nourri les préjugés coloniaux, tel celui que les Africains ne peuvent pas administrer leurs affaires eux-mêmes (Dunn 109). Il suffit de voir les réponses de l'auteur à la question qu'il se pose : «Le Noir, un incapable ?» (25). Ce récit qui suit, est fort informatif. En 1989, le chef des Services Secrets sud-africains se rend au Zaïre. L'auteur fait partie du cortège qui accompagne la délégation. La traversée de la ville et des misères qu'elle étale, est source d'angoisse ; tout le monde s'efforce à distraire les invités pour qu'ils ne se laissent attirer par le spectacle « honteux » de la pauvreté. Cette angoisse est ainsi expliquée :

Après leur départ, nous nous demandons s'ils ne seront pas confortés dans leur conviction que le Noir est un bon à rien, tout juste capable, tel un petit enfant, de détruire le coûteux jouet que viennent de lui offrir ses parents, idée qui serait dommageable pour nos frères du pays de l'apartheid, et plus encore pour Mandela dont la libération risque d'être repoussée indéfiniment. (26)

Ces sentiments d'infériorité et de culpabilité déterminent à bien des égards les propos de l'auteur. La psyché du corrompu est triomphale; la victime est désarmée et rejoint le bourreau dans un rapport où tout est perverti, même la valeur de la vie, devenue un bien comme un autre comme le démontre, par exemple, le massacre des étudiants du campus de Lumumbashi en 1990 :

Les familles des dizaines d'étudiants natifs de la province qui ont été assassinés le 11-12 mai ont reçu d'importantes sommes d'argent pour ne pas porter le deuil de leurs enfants! Les militaires sont également récompensés pour leur 'bravoure'. Tout ce monde se rue sur les boutiques qui sont vidées. Les prix flambent... (40)

Les regards étrangers ordonnent la narration et l'homogénéisent en agissant en intensificateurs de continuités morales qui viennent combler le déficit moral engendré par la corruption.

La nostalgie de l'ère coloniale, déjà évoquée, est symptomatique d'une absence totale de tout projet historique. Ce fond nihiliste conduit à une amnésie volontaire. 
Pourtant, il suffit de chercher dans l'histoire même du pays pour trouver des ingrédients susceptibles de remodeler sa destinée. L'indice le plus révélateur est l'absence de toute référence au lomumbisme. Cet effacement met le livre entier dans une logique qui, face à l'État corrompu, trouve dans la domination coloniale le paradis perdu.

Au cynisme propre au mobutisme, Dungia oppose un discours essaimé de vérités dont la validation dépend parfois d'acteurs qui contredisent la substance éthique qu'il assigne à sa mission. Le discours du contre-pouvoir a besoin de s'appuyer sur des alliés, parfois de tout vent.

La représentation de la corruption sert, en réalité, de prétexte à l'auteur pour souligner son propre rôle dans l'affirmation de l'identité du groupe. L'argument est soumis à l'autorité. Écrire obéit, tout d'abord, au désir d'affirmer une appartenance et un rôle. Derrière l'idée de servir une certaine vérité, se cache le besoin de revendiquer un statut. La conception de l'identité est soumise à une logique binaire qui fait que l'autre corrompu représente tout ce qui s'oppose aux valeurs dont l'auteur se considère le porteparole. Par conséquent, la représentation tombe dans le piège essentialiste. La biographie de Mobutu ne fait que confirmer les valeurs et rôles défendus par l'auteur. Du portrait de l'ancien président zaïrois sont éliminés tous les éléments susceptibles de contredire un tel projet.

L'idée d'une autorité érigée en apriori ne définit pas seulement la représentation, mais aussi le rapport au lecteur. En dévoilant les déviances du régime, l'auteur suppose la naissance d'un pacte avec le lecteur. Dungia entre dans un rapport d'identité avec ce dernier : Je est autre sachant que tous les deux adhèrent aux mêmes valeurs.

Le discours sur la corruption a comme objectif d'assurer le consensus; il est destiné à ces «30 millions d'êtres affamés dans un pays gorgé de richesses » (30), ces citoyens d'un «paradis confisqué » (26) où vivent 99\% d'une population démunie (22). En révélant les secrets de la dictature, l'auteur veut créer un pouvoir fédérateur autour d'une certaine vérité. Le pouvoir de son savoir, tout paradoxalement qu'il soit comme nous l'avons tenté de le démontrer, réside dans sa capacité à servir de voix à la majorité écrasée. Les révélations mises en circulation ont la même fonction que la monnaie : elles n'ont de valeur que parce qu'elles circulent. Et, comme la monnaie, elles déterminent les rapports entre les membres d'un groupe. 


\section{Ouvrages cités}

Angulu, M. Edi. Adieu Mobutu «Génie » de Gbadolite. De la ditature authentique à la démocratie intégrale. Préface René Dumont. Genève : DS, 1991.

Bertrandias, Bernadette, dir. Le secret. Clermont-Ferrand : Centre de recherches sur les littératures modernes et contemporaines, PU Blaise Pascal, 1999.

Braeckman, Colette. Le dinosaure. Le Zä̈re de Mobutu. Paris : Fayard, Librairie Arthème, 1992.

Broglie, Duc de. Le secret du roi. Correspondance secrète de Louis XV avec ses agents diplomatiques (1752-1774). Paris : Calmann Lévy, deuxième édition, 1878.

Coquet, Jean-Claude. Le discours et son sujet (essai de grammaire modale). Tome 1. Paris : Klinckieck, 1984.

Diangitukwa, Fweley. Marechal Mobutu, je vous parle. Saint-Léger : Afrique Nouvelle, 1990.

Duke, Lynn. Mandela, Mobutu, and me : A Newswoman's African Journey. New York : Doubleday, 2003.

Dungia, Emmanuel. Mobutu et l'argent du Zä̈re. Révélations d'un diplomate, ex-agent des services secrets. Paris : L'Harmattan, 1992.

Dunn, Kevin. Imagining the Congo : The International Relations of Identity. New York : Palgrave MacMillan, 2003.

Emizet, Kisangani N. F. Zaire after Mobutu. A Case of a Humanitarian Emergency. UNU World Institute for Development Econonomics Research, 1997.

Haskin, Jeanne M. The Tragic State of the Congo : From Decolonization to Dictatorship. New York : Algora, 1994.

Lanotte, Olivier. Guerres sans frontières en République Démocratique du Congo. Bruxelles : Grip-Editions Complexe, 2003.

Leslie, Winsome J. Zaire: Continuity and Political Change in an Oppressive State. Boulder : Westview, 1991.

Oyatambwe, Wamu. De Mobutu à Kabila. Avatars d'une passation inopinée. Paris: L'Harmattan, 1999.

Remilleux, Jean-Louis. Mobutu. Dignité pour l'Afrique. Entretiens avec Jean-Louis Remilleux. Paris : Albin Michel, 1989. 
Schatzberg, Michael. Mobutu or Chaos ? The United States and Zaire, 1960-1990. Lanham : UP of America, 1991.

Woodiwiss, Michael. Gangster Capitalism. The United States and the Global Rise of

Organized crime. New York : Carroll \& Graf, 2005.

\section{Notes}

${ }^{1}$ À titre d'exemple, ces révélations sur une société secrète à laquelle Mobutu adhère: «Le détail du 'rite' Cissé m'a été révélé par un garde du corps et par un membre de la suite présidentielle chargé de certaines missions ultra-secrète du Guide » (47) ou sur cette opération-démasquage : «M. Kengo est discret comme un Sioux. On ne lui connaît aucune affaire au Zaïre. J'en découvre pourtant une au cours de mes relations professionnelles avec le représentant du bureau de Liaison de l'Afrique du Sud, M. Hanekon » (77).

${ }^{2}$ Sur la nature de la structure policière et son service au régime, voir Braeckman, Emizet, Haskin, Schatzberg, Woodiwiss.

${ }^{3}$ Braeckman présente le secret comme une arme qui mine toute causalité et, de ce fait, libère le sujet des conséquences de ses actes. Il décrit Mobutu comme quelqu'un qui tient ses distances et fausse systématiquement les cartes pour éviter toute implication directe dans des crimes commis : «Mobutu c'est le tueur aux mains propres. Il conçoit les crimes, mais ne les exécute pas » (31).

${ }^{4}$ Dans un autre contexte, le Duc de Broglie ne cesse pas d'annoncer son inquiétude face au contenu non encore complètement divulgué de ce qu'il qualifie de « diplomatie secrète » de Louis XV : « Mais qu'est-ce que cette mystérieuse correspondance? Qu'était-ce que cette affaire secrète, ce secret du Roi, comme on l'appelle habituellement dans les documents contemporains? Quels en étaient le but et l'objet ? Quels en furent les confidents? Quelle action fut exercée par cette diplomatie clandestine sur les événements politiques de cette triste époque ? » (ii). Même en décrivant le roi comme une personne « débile », l'auteur ne cache pas son inquiétude vis-à-vis des recoins du secret non encore explorés. Chaque question est un acte politique dans la mesure où elle cherche à dévoiler le pouvoir (la découverte étant ici conquête).

${ }^{5}$ Sur la politique de pillage systématique par Mobutu et son entourage, voir Emizet.

${ }^{6}$ Pour Bertrandias, « Les discours tissés autour des secrets [...] n'aboutissent sans doute qu'à de vertueux mensonges, l'inscrutable ne dévoilant toujours que sa parodie, les secrets n'étant que des postiches visant à voiler / à dire, la face nue / indicible, du mystère » (5).

${ }^{7}$ Voir aussi Leslie, 43.

${ }^{8}$ Sur comment le manque de légitimité rend le pouvoir dépendant de puissances extérieures, notamment les Etats-Unis, voir Schatzberg.

${ }^{9}$ Voir aussi Schatzberg, 52.

${ }^{10}$ Mobutu veut donner de lui-même l'image de celui sans qui le Zaïre ne peut exister. Cette idée est largement défendue (non seulement dans le cas de Mobutu, mais chez bien d'autres dictateurs), le remarque Remilleux : «Le Congo avait été belge avec tout ce que cela implique, puis indépendant et englué dans un magma politique d'où émergea Patrice Lumumba, puis attaqué de l'intérieur par les sécessionnistes souvent manipulés, pour devenir enfin le Zaïre, pays, fleuve, monnaie, nation créée et façonnée par un homme, Mobutu, qui galvanisa les consciences, pacifia cet immense territoire, donnant un espoir formidable à tout un peuple jusque-là déchiré par les guerres tribales » (9-10).

${ }^{11}$ D'ailleurs, c'est cette identification avec le lecteur virtuel qui implicitement donne son statut à Dungia en tant qu'énonciateur de vérités immaculées; d'où le manque de besoin de se justifier même s'il faisait partie de la haute bureaucratie au temps du mobutisme. Énoncer des secrets innocents.

${ }^{12}$ Voir sur ce point Angulu, 95-98.

${ }^{13}$ Voir Oyatambwe, 12-28.

${ }^{14}$ Pour plus de détails, voir Braeckman, 13-27. 